\title{
A new rank-ordered method for removing random-valued impulse noise
}

\author{
Hao Yang ${ }^{1, a}$ and Leiting Chen ${ }^{1, b}$ \\ ${ }^{1}$ School of Computer Science and Engineering, University of Electronic Science and Technology of \\ China, Chengdu, Sichuan 611731, P.R.China \\ avhaoyang@gmail.com, ${ }^{b}$ richardchen@uestc.edu.cn
}

Keywords: Random-valued impulse noise, Sub-neighborhood mean, Rank-ordered extension differences, Edge-preserving regularization.

Abstract. In this paper, we present an effective statistic algorithm to remove the random-valued impulse noise. The proposed method takes full advantage of the sub-neighborhood mean of each pixel to reduce the missed and failed detection in high noise ratio. In the first phase, a statistic based on rank-ordered extension differences is defined to identify pixels which are noise candidates. In the second phase, all noisy pixels will be restored by an edge-preserving regularization filter. Simulation results show that the proposed method has better accuracy than many other existing techniques with high noise level.

\section{Introduction}

Digital images are frequently deteriorated by impulse noise during their acquisition, storage, transmission or analog-to-digital conversion [1]. The corrupted images could hamper the subsequent image processing operation, such as image segmentation and object recognition. Therefore, it is very fundamental and important to eliminate noise in the images before subsequent.

A number of denoising methods have been proposed to remove the impulse noise distributions in the past. The median filter (MED) [2], the weighted median (WM) filter [3] and the center weighted median filter (CWM) [4] are widely applied for their simplicity and high computational efficiency. However, they implement uniformly across the whole image without distinguishing whether a pixel is noise or not. Thus, a two-phase strategy which detects noise before filtering has been presented, such as the Switching median (SWM) filter [5], the adaptive center-weighted median (ACWM) filter [6], directional weighted median (DWM) filter [7] and the adaptive switching median (ASWM) filter [8]. In [9], a local statistic rank-ordered absolute difference (ROAD) was presented. Next, Dong et al. presented a more robust statistic rank-ordered logarithmic difference (ROLD) [10] based on ROAD and combined it with the edge-preserving regularization method (EPR) [11] for suppressing random-valued impulse noise while preserving the detail.

In this paper, we propose a statistic algorithm based on improved ROAD and EPR for removing random-valued impulse noise. The proposed method takes full advantage of the sub-neighborhood mean of each pixel to reduce the missed and failed detection in high noisy ratio. Simulation results show that our method performs better accuracy than other techniques even at high noise levels.

\section{Our Method}

Extension differences. The ROAD statistic is effective but is sensible to the proportion of the impulse noise. It is easy to get error detection when noise ratio is high. In order to overcome the drawback, we improve the absolute differences of ROAD by using the sub-neighborhood mean instead of the absolute value.

In ROAD, the processing pixel is the central one, and its surrounding pixels are used to compute the absolute differences. We define a sub-neighborhood for each pixel which surrounding the processing pixel.

For $3 \times 3$ sliding window, the processing pixel $(i, j)$ is in the center and eight pixels $(k, l)$ surround it. See below Fig. 1 


\begin{tabular}{|c|c|c|c|c|}
\hline & & & \\
\hline( & $(k, 1)$ & $(k, j)$ & $(k, 1)$ & \\
\hline & $-(k, 1)-$ & $\langle i, j\rangle$ & $(k, 1)$ & \\
\hline & $(k, 1)$ & $(k, 1)$ & $(k, 1)$ & \\
\hline & & & & \\
\hline
\end{tabular}

Fig. 1 Sub-neighborhood

We marked a sub-neighborhood with ellipse in this figure. There are eight sub-neighborhoods which are defined as follows:

$$
\begin{aligned}
& \Omega_{k, l}^{1}=\left\{y_{i+s, j+t}:-2 \leq s \leq 0,-2 \leq t \leq 0\right\}, \\
& \Omega_{k, l}^{2}=\left\{y_{i+s, j+t}:-2 \leq s \leq 0,-1 \leq t \leq 1\right\}, \\
& \Omega_{k, l}^{3}=\left\{y_{i+s, j+t}:-2 \leq s \leq 0,0 \leq t \leq 2\right\}, \\
& \Omega_{k, l}^{4}=\left\{y_{i+s, j+t}:-1 \leq s \leq 1,-2 \leq t \leq 0\right\}, \\
& \Omega_{k, l}^{5}=\left\{y_{i+s, j+t}:-1 \leq s \leq 1,0 \leq t \leq 2\right\}, \\
& \Omega_{k, l}^{6}=\left\{y_{i+s, j+t}: 0 \leq s \leq 2,-2 \leq t \leq 0\right\}, \\
& \Omega_{k, l}^{7}=\left\{y_{i+s, j+t}: 0 \leq s \leq 2,-1 \leq t \leq 1\right\}, \\
& \Omega_{k, l}^{8}=\left\{y_{i+s, j+t}: 0 \leq s \leq 2,0 \leq t \leq 2\right\} .
\end{aligned}
$$

Each sub-neighborhood or set has a mean $M_{k, l}^{h}$ defined as:

$$
M_{k, l}^{h}=\operatorname{mean}\left(\Omega_{k, l}^{h}\right), h \in\{1,2, \ldots, 8\} .
$$

Then, we define the extension differences $\tilde{d}(i, j)$ by computing the difference between the processing pixel $(i, j)$ value and the mean $M_{k, l}^{h}$ of each sub-neighborhood. It is expressed below:

$$
\tilde{d}(i, j)=\left|M_{k, l}^{h}-y_{i, j}\right|
$$

ROED detector. Based the extension differences, we can define a rank-ordered statistic to detect pixels. The statistic is named rank-ordered extension differences (ROED).

Like ROAD, to sort the values of $\tilde{d}(i, j)$ in increasing order, and let $\tilde{r}_{n}(i, j)$ denote the $\mathrm{n}^{\text {th }}$ smallest one in ordered queue. The ROED statistic can be expressed:

$$
\operatorname{ROED}(i, j)=\sum_{n=1}^{m} \tilde{r}_{n}(i, j)
$$

Then, we define the detector as follows:

$$
D e t=\left\{\begin{array}{l}
1, \text { if } R O E D>T ; \\
0, \text { Otherwise, }
\end{array}\right.
$$

where $\mathrm{T}$ is a threshold. If the ROED value of a pixel is greater than the threshold, the pixel would be labeled as noise-like. Otherwise, the pixel is noise-free. 
A two-phase filter based on ROED and EPR. After having a good detector, we can combine it with the edge-preserving regularization [11] in a two-phase method.

To ensure a high accuracy of detection, it is executed iteratively with decreasing thresholds, see [12]. At early iterations, large thresholds will identify pixels that are most likely to be noisy. In the subsequent iterations, the threshold is decreased to include more noise candidates. The iteration threshold $T^{t}$ is defined as follows:

$$
T^{t}= \begin{cases}T^{0}, & t=0 ; \\ 0.8 T^{t-1}, & t>0,\end{cases}
$$

where $t$ is the number of iterations.

\section{Experimental Results}

In this section, the proposed algorithm is compared with many existing techniques. Extensive experiments are conducted on the classic $512 \times 512$ gray scale image, Lena.

Noise Detection. A good noise detector should be able to keep the rate of both miss-detection and false-detection at a low level. We combine them into miss-false detection indicator. Hence, we compare our proposed detector with ROAD [9] and ROLD [10] detectors by using the rate of miss-false detection. The result is shown in Fig. 2

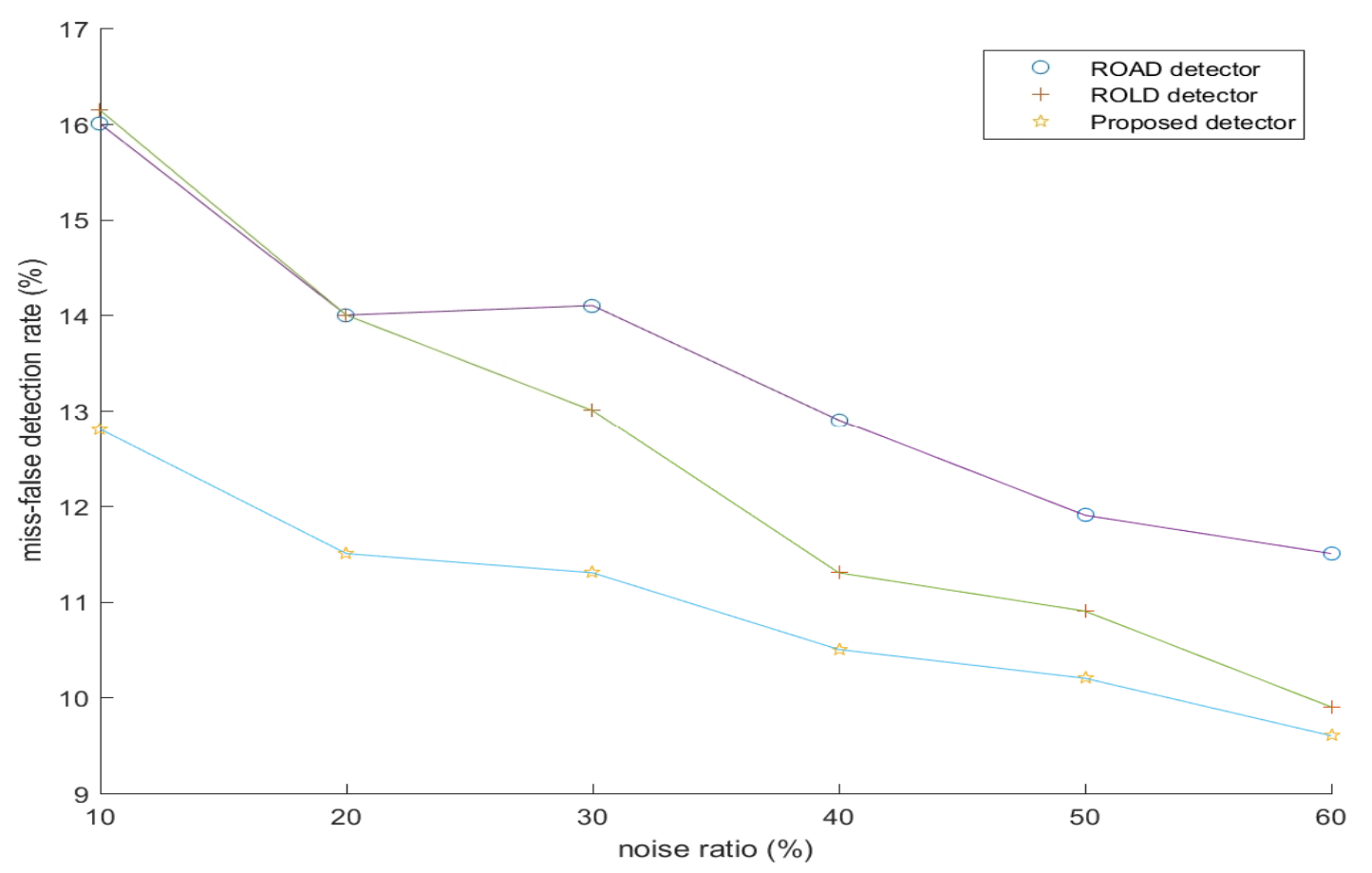

Fig. 2 Miss-false detection rate

In this figure, our method has the lowest miss-false detection rate for all noise level. Especially in low noise case, our method has a great improvement on detection accuracy. The reason is that the mean of sub-neighborhood can effectively suppress the influence which noise act on the rank statistic.

Image Restoration. Restoration results are quantitatively compared by using the peak signal-to-noise ratio (PSNR). Table 1 lists the PSNR values obtained from various methods for the image with noise densities from $20 \%$ to $60 \%$. The best values are marked in boldface. From the table, it is observed that our method generates the best results for all noise ratios. 
Table 1 Comparison of restoration results in PSNR

\begin{tabular}{|c|c|c|c|}
\hline \multirow{2}{*}{ Algorithm } & \multicolumn{3}{|c|}{ Noise ratio } \\
\cline { 2 - 4 } & $20 \%$ & $40 \%$ & $60 \%$ \\
\hline SMF & 31.20 & 27.75 & 22.66 \\
\hline ACWM & 36.07 & 28.79 & 21.19 \\
\hline ROAD-TRIF & 36.70 & 31.12 & 26.08 \\
\hline ROLD-EPR & 37.45 & 32.76 & 29.03 \\
\hline Proposed & $\mathbf{3 7 . 8 8}$ & $\mathbf{3 2 . 9 7}$ & $\mathbf{2 9 . 1 1}$ \\
\hline
\end{tabular}

\section{Conclusions}

In this paper, an effective algorithm is proposed for removing random-valued impulse noise by two-phase filter based on ROED and EPR. Simulation results show that our method performs better accuracy than other techniques even at high noise levels.

\section{References}

[1] R. Gonzalez and R. Woods: Digital image processing. Prentice Hall, 2002, 190.

[2] W. Pratt: Median filtering tech rep. ... Proc Institute. Los Angeles: Univ of Southern California, 1975.

[3] D.R. K. Brownrigg: The weighted median filter. Communications of the ACM, vol. 27 (1984), p. 807-818.

[4] S.J. Ko and Y.H. Lee: Center weighted median filters and their applications to image enhancement. IEEE Transactions on Circuits and Systems, vol. 38 (1991), p. 984-993.

[5] T. Sun and Y. Neuvo: Detail-preserving median based filters in image processing. Pattern Recognition Letters, vol. 15 (1994), p. 341-347.

[6] T. Chen and H.R. Wu: Adaptive impulse detection using center-weighted medial filters. IEEE Signal Processing Letters, vol. 8 (2001), p. 1-3.

[7] Y.Q. Dong and S.F. Xu: A new directional weighted median filter for removal of random-valued impulse noise. IEEE Signal Processing Letters, vol. 14 (2007), p. 193-196.

[8] S. Akkoul, R. Léedée, R. Leconge, and R. Harba: A new adaptive switching median filter. IEEE Signal Processing Letters, vol. 17 (2010), p. 587-590.

[9] R. Garnett, T. Huegerich, C. Chui, and W.J. He: A universal noise removal algorithm with an impulse detector. IEEE Transactions on Image Processing, vol. 14 (2005), p. 1747-1754.

[10] Y.Q. Dong, R.H. Chan, and S.F. Xu: A detection statistic for random-valued impulse noise. IEEE Transactions on Image Processing, vol. 16 (2007), p. 1112-1120.

[11] M. Nikolova: A variational approach to remove outliers and impulse noise. Journal of Mathematical Imaging and Vision, vol. 20 (2004), p. 99-120.

[12] R.H. Chan, C.W. Ho, C.Y. Leung, and M. Nikolova: Minimization of detail-preserving regularization functional by Newton's method with continuation. Proceedings - International Conference on Image Processing, ICIP, vol. 1 (2005), p. 125-128. 